\title{
Heritability of Transforming Growth Factor- $\beta 1$ and Tumor Necrosis Factor-Receptor Type 1 Expression and Vitamin D Levels in Healthy Adolescent Twins
}

\author{
Natalie T. Mills, ${ }^{1,2}$ Margie J. Wright, ${ }^{1}$ Anjali K. Henders, ${ }^{1}$ Darryl W. Eyles, ${ }^{2,3}$ Bernhard T. Baune, ${ }^{4}$ \\ John J. McGrath, ${ }^{2,3}$ Enda M. Byrne, ${ }^{2}$ Narelle K. Hansell, ${ }^{1}$ Eva Birosova, ${ }^{5}$ James G. Scott, ${ }^{6}$ \\ Nicholas G. Martin, ${ }^{1}$ Grant W. Montgomery, ${ }^{1}$ Naomi R. Wray, ${ }^{2}$ and Anna A. E. Vinkhuyzen ${ }^{2}$ \\ ${ }^{1}$ Genetic Epidemiology, OIMR Berghofer Medical Research Institute, Brisbane, Queensland, Australia \\ ${ }^{2}$ Queensland Brain Institute, University of Queensland, Brisbane, Queensland, Australia \\ ${ }^{3}$ Queensland Centre for Mental Health Research, The Park Centre for Mental Health, Brisbane, Queensland, Australia \\ ${ }^{4}$ Discipline of Psychiatry, School of Medicine, University of Adelaide, Adelaide, South Australia, Australia \\ ${ }^{5}$ School of Medicine, James Cook University, Townsville, Queensland, Australia \\ ${ }^{6}$ Metro North Mental Health, Royal Brisbane and Women's Hospital, Brisbane, Queensland, Australia
}

\begin{abstract}
Cytokines and vitamin D both have a role in modulating the immune system, and are also potentially useful biomarkers in mental illnesses such as major depressive disorder (MDD) and schizophrenia. Studying the variability of cytokines and vitamin $D$ in a healthy population sample may add to understanding the association between these biomarkers and mental illness. To assess genetic and environmental contributions to variation in circulating levels of cytokines and vitamin D (25-hydroxy vitamin D: 25(OH)D3), we analyzed data from a healthy adolescent twin cohort (mean age 16.2 years; standard deviation 0.25). Plasma cytokine measures were available for 400 individuals ( $85 \mathrm{MZ}, 115 \mathrm{DZ}$ pairs), dried blood spot sample vitamin D measures were available for 378 individuals (70 MZ, $118 \mathrm{DZ}$ pairs). Heritability estimates were moderate but significant for the cytokines transforming growth factor- $\beta 1$ (TGF- $\beta 1), 0.57$ (95\% Cl 0.26-0.80) and tumor necrosis factor-receptor type 1 (TNFR1), 0.50 (95\% $\mathrm{Cl} 0.11-0.63$ ) respectively. Measures of 25(OH)D3 were within normal range and heritability was estimated to be high $(0.86,95 \% \mathrm{Cl} 0.61-0.94)$. Assays of other cytokines did not generate meaningful results. These potential biomarkers may be useful in mental illness, with further research warranted in larger sample sizes. They may be particularly important in adolescents with mental illness where diagnostic uncertainty poses a significant clinical challenge.
\end{abstract}

Keywords: cytokines, vitamin D, major depression, psychosis, heritability

Emerging evidence suggests a range of blood measures may be useful biomarkers in mental health disorders such as MDD and schizophrenia. Potential blood biomarkers include measures of vitamin D, cytokines, C-reactive protein (CRP), and antibodies to infectious agents (Arias et al., 2012; Howren et al., 2009; Tolppanen et al., 2012). Blood biomarkers may be particularly relevant in adolescence, where depression is often comorbid with other disorders (Garber \& Weersing, 2010), resulting in diagnostic uncertainty. Genetic and environmental influences both contribute to the etiology of psychiatric disorders (Kendler, 2013). Heritability is estimated around 40\% for MDD (Glowinski et al., 2003; Sullivan et al., 2000) and around $80 \%$ for schizophrenia (Schwab \& Wildenauer, 2013). The presence of plasma pro-inflammatory cytokines (Gabbay et al., 2009; Mills et al., 2013) and variation in vitamin D levels (Tolppanen et al., 2012) in adolescents with mental illness is especially interesting, with both also having a role in the modulation of the immune system (Yin \& Agrawal, 2014). Understanding how putative biomarkers vary in a healthy population of adolescents may help in guiding the study of these blood measures in psychiatric disorders in adolescents. Examining the link between psychiatric disorders and immune system dysregulation (Gabbay et al.,

RECEIVED 26 August 2014; ACCEPTED 3 September 2014. First published online 3 December 2014.

ADDRESS FOR CORRESPONDENCE: Dr Natalie Mills, Genetic Epidemiology, QIMR Berghofer Medical Research Institute, Herston QLD 4006, Australia. E-mail: natalie.mills@ qimrberghofer.edu.au 
2009), and the genetic contribution to these biomarkers, are both required to understand the role of plasma cytokines and vitamin D levels in adolescent psychiatric disorders.

\section{Cytokines}

Cytokines are small proteins that mediate key steps in cellular and humoral immunity (Janssen et al., 2010), and have been shown to act as neuromodulators. Examples of proinflammatory cytokines include interleukin-1 $\beta$ (IL-1 $\beta$ ), interleukin-6 (IL-6), and tumor necrosis factor- $\alpha$ (TNF- $\alpha$ ) (Dowlati et al., 2010; Miller et al., 2011), with TNFR1 the key mediator of TNF signaling in most cells (Wajant et al., 2003). Some cytokines, such as transforming growth factorbeta (TGF- $\beta$ ) and TGF- $\beta 1$, have both pro-inflammatory and anti-inflammatory effects (Gressner et al., 2002; Wahl, 1992).

Cytokines, in particular pro-inflammatory cytokines, have been associated with both MDD and schizophrenia. MDD is thought to be associated with immune system dysregulation (Danese et al., 2007; 2011; Gabbay et al., 2009; Irwin \& Miller, 2007). In adults, a recent meta-analysis reported an association between elevated levels of two pro-inflammatory cytokines, IL- 6 and TNF- $\alpha$, and MDD (Dowlati et al., 2010). It is also possible that symptoms of depression may be a result of natural variation between individuals in levels of circulating cytokines, even in the absence of specific immune challenge such as infection.

A role for immune system dysregulation in schizophrenia has also been hypothesized (Benros et al., 2012; Ripke et al., 2014). Levels of circulating cytokines have been associated with acute exacerbations of schizophrenia. Specifically, a recent meta-analysis reported an increase in the cytokines IL-1 $\beta$, IL-6, and TGF- $\beta$ during acute relapse and/or first episode psychosis, which normalized with antipsychotic treatment (Miller et al., 2011). The same meta-analysis found IL-12, TNF- $\alpha$, Interferon- $\gamma$ (IFN- $\gamma$ ), and soluble IL2 receptor (sIL-2R) appeared to be trait markers, with levels elevated in first episode psychosis, and remaining so following antipsychotic treatment. Levels of IL-12, TNF- $\alpha$, and IFN- $\gamma$ were also found to be elevated in acute relapses of schizophrenia, and remained elevated following antipsychotic treatment (Miller et al., 2011).

Some studies have measured cytokine response in stimulated cells; for example, ex vivo stimulation with amyloid$\beta$ in whole blood (Posthuma et al., 2005), or antigenstimulated whole-blood assays (Stein et al., 2003). This is likely to reflect the heritability of the immune response rather than natural variation. Few studies have estimated the heritability of circulating levels of cytokines, particularly in adolescents. Heritabilities of circulating levels of the cytokines IL-1 $\beta$, IL-6, IL-10, and TNF- $\alpha$ have been estimated in adult females, with the cytokines IL- $1 \beta$ and IL-10 found to be moderately heritable (range $0.27-0.32$ and 0.3 for IL-1 $\beta$ and IL-10, respectively), and the cytokines IL6 and TNF- $\alpha$ found to be less heritable (range 0.15-0.16 and $0.17-0.23$ for IL- 6 and TNF- $\alpha$, respectively; Sas et al., 2012). It should also be noted that measurement of cytokines is known to present difficulties, and quantification of cytokine levels has been found to depend on a number of factors. For example, concentrations of several inflammatory markers have been found to change depending on whether serum or plasma is collected (Skogstrand, 2012), and healthy adolescents should have very low levels of circulating inflammatory cytokines (Miller et al., 2011). Furthermore, circulating levels of some cytokines vary throughout a 24-hour period (e.g., IL-2; Palm et al., 1996), or a seven-day period (e.g., TNF- $\alpha$; O'Brien et al., 2007). These challenges all need to be considered in the design of studies involving the measurement of cytokines.

\section{Vitamin D}

Vitamin D is best known for its role in skeletal health. More recently, vitamin $\mathrm{D}$ has also been implicated in non-skeletal health such as cancers, immunology, and psychiatric disease (Cantorna, 2012; Holick, 2007; Yin \& Agrawal, 2014). Activation of cells of the immune system results in upregulation of the vitamin D receptor (Veldman et al., 2000). Furthermore, vitamin $\mathrm{D}$ is a direct inhibitor of the proinflammatory cytokine IL-17 (Bruce et al., 2011). With regard to psychiatric disease, an increased risk of depression has been associated with low vitamin D in cross-sectional studies (Hoang et al., 2011), yet this may reflect individuals with depression spending less time outdoors with less sun exposure. However, a prospective study of 2,759 individuals found a significant association between low vitamin D measured at 9 years of age, and higher scores on depressive symptoms at 11 and 14 years of age (Tolppanen et al., 2012). With regard to schizophrenia, an association has been observed with increased risk of this syndrome later in life in neonates who had either low levels or high levels of vitamin D (McGrath et al., 2010).

Heritability estimates for vitamin $\mathrm{D}$ show much variation and some also seem to vary across season of measurement and sex. Heritability of vitamin D ranges from 0.43 in European adult twins ( $98.3 \%$ of these twins were female; Hunter et al., 2001) to as high as 0.80 in the German Asthma Family Study Group (Wjst et al., 2007). A study of male twins found vitamin D levels to be highly heritable during winter, but not heritable in summer (Karohl et al., 2010), while a study of male and female twins found vitamin $\mathrm{D}$ to be heritable in summer, but not during winter (Snellman et al., 2009). Sex differences have been observed with a study of vitamin D in rural Chinese adolescent twins estimating heritability at 0.86 for males and 0.17 for females (Arguelles et al., 2009).

With the clinical diagnosis of both MDD and schizophrenia likely to represent a biological heterogeneous group of disorders, identification of biomarkers that stratify patients for application of 'stratified medicine' is a direction worthy of further research (Kapur et al., 2012). The study reported here represents preliminary research towards the 
goal of understanding the role of potential blood biomarkers (specifically plasma cytokine and vitamin D measures) of depression and psychosis. We could not find prior published heritability estimates for some of these cytokines. We used a population-based sample of healthy adolescent twins to assess the genetic and environmental contributions to variation in circulating levels of these potential biomarkers.

\section{Methods}

\section{Samples}

Participants were 16-year-old twins (mean 16.2 years, SD 0.25 years) from the Brisbane adolescent twin study (Wright \& Martin, 2004). In this sample, both plasma cytokine and dried blood spots (DBS) vitamin D measures were collected as part of a study focusing on health and well-being in an adolescent population sample. Plasma cytokine measures were available for 400 individuals ( $85 \mathrm{MZ}$ twin pairs and 115 DZ twin pairs), DBS vitamin D measures were available for 378 individuals $(70 \mathrm{MZ}$ twin pairs and $118 \mathrm{DZ}$ twin pairs, two twin pairs were incomplete). Of these twin pairs, $49 \mathrm{MZ}$ twin pairs and $74 \mathrm{DZ}$ twin pairs had data available for both plasma cytokine and vitamin D measures.

Blood was collected when twins came in for testing as close as possible to their 16th birthday. To explore impact of age of biological sample in this pilot study we selected individuals who participated either between the years 1997-2000 (wave 1) or between the years 2006-2009 (wave 2). Blood samples were available from these years both as plasma (for measurement of cytokines) and DBS (for measurement of vitamin D). DBS for the measurement of vitamin D were stored on Whatman 903 filter paper for wave 1, and on Whatman FTA cards impregnated with antibacterial agents to preserve DNA for wave 2. Blood of two members of a twin pair (i.e., co-twins) was always collected within the same wave.

\section{Assay of Plasma Cytokine and Vitamin D Measures}

Plasma cytokine measures. A total of 25 plasma cytokines and inflammatory markers were measured at the James Cook University, Townsville, Australia. Of these 25 markers, TGF- $\beta 1$ and TNFR1 were assayed successfully. Specifically, plasma cytokines and inflammatory markers were divided into four separate groups, based on multiplexing compatibility with other cytokines and inflammatory markers with the groups as follows: Group 1: TGF- $\beta 1$; Group 2: interleukin-2 (IL-2), IL-4, IL-7, IL-11, IL-12, IL-13, TNF- $\alpha$, IL-17A, IL-21, Exotaxin, Granulocyte Macrophage ColonyStimulating Factor (GM-CSF); Group 3: IL-1 $\alpha$, IL-1 $\beta$, IL3, IL-5, IL-6, IL-8, IL-9, IL-10, IFN- $\gamma$-inducible protein 10 (IP-10), Monocyte Chemo-attractant protein-1 (MCP1), Macrophage inflammatory protein-1a (Mip-1a), IFN- $\gamma$; Group 4: TNFR1.

TGF- $\beta 1$ and TNFR1 were measured individually (not part of the multiplex) due to not being compatible with other cytokines. For TGF- $\beta 1$ (560429) we used the human TGF- $\beta 1 D$ uoset ELISA kit (R\&D). We used BD biosciences flex set multiplex assays at half strength (allowing double the number of samples) compared to the manufacturer's recommendations, supported by pilot analyses. Plasma samples were diluted four times before testing, consistent with observations from the kit manufacturer (and our own preexperiment measurements) that undiluted plasma causes high background signal that interferes significantly with specific signals. Standard curves were generated using technical controls of lyophilized standard protein at a known concentration. In addition, for all cytokines and inflammatory markers an intra-assay experiment (to evaluate accuracy of the method) was run on 20 samples created from tissue culture medium spiked with standards. The experimental design for the plasma samples ensured that co-twins (two members of a twin pair) were randomized across plates.

Out of the cytokines measured in plasma, we found that only the two assays conducted for individual cytokines (TGF- $\beta 1$ and TNFR1) not included in the multiplex generated meaningful results. For the multiplexed cytokines (groups 2 and 3), we found that although the technical controls (the intra-assay experiment) were measured accurately, the assay was not sufficiently sensitive to the low circulating levels in healthy adolescents following the recommended dilution of sample.

Given the poor outcome of cytokine assays from plasma samples, we also attempted to assay cytokines from DBS (Skogstrand et al., 2008). Inflammatory markers are more stable in DBS, with measurable concentrations of cytokines found to be stable in DBS stored at different temperatures for many days when compared to DBS frozen immediately after preparation (although long-term storage at $-20{ }^{\circ} \mathrm{C}$ is considered optimal; Skogstrand et al., 2008). While measurable amounts of most cytokines have been found to be nearly constant in DBS stored up to 23 years, a marked decrease over time has been noted in measurable concentrations of IL-1 $\beta$, IL-8, sIL-6ra, matrix metallopeptidase9 (MMP-9), triggering receptor expressed on myeloid cells 1 (TREM-1), CRP, brain-derived neurotrophic factor (BDNF), and neurotrophin-4 (NT-4) in stored DBS (Skogstrand et al., 2005). We found that the cytokine measures were sensitive to paper type (often generating null reads for one or other paper) and were subject to plate effects that could not be adjusted appropriately in an experimental design in which co-twins within a twin pair were present on the same plate. We also attempted to measure antibodies to infectious agents (Yolken et al., 2011), another potential biomarker for MDD, but also found the assays to be highly sensitive to paper type and plate effects, and few individuals had non-null measures.

Vitamin D measures. The main circulating form of vitamin D is 25 hydroxyvitamin D3 (25(OH)D3) (Holick, 2007). In our study, 25(OH)D3 was measured from DBS by 
liquid chromatography tandem mass spectroscopy (Eyles et al., 2009; McGrath et al., 2010) at the University of Queensland, Brisbane, Australia. The assay method is highly sensitive, and can measure 25(OH)D3 in DBS stored for between 8 and 22 years (Eyles et al., 2009). With this method, assay calibrants are extracted and derived in the same way as clinical samples, using multi-point calibration curves (Eyles et al., 2009). Co-twins were plated in adjacent wells on the same plate. As Whatman FTA paper is thicker than 903 and therefore absorbs more blood, standards were prepared on both paper types in accordance with previous studies (Kvaskoff et al., 2012).

For the measurement of $25(\mathrm{OH}) \mathrm{D} 3$, the experimental design for the DBS was not ideal for partitioning of variance as co-twins of the same pair were plated in neighboring wells of the same plate. Under this design, batch effects are common to the twin pair so will be partitioned into the common environmental component. In contrast, if co-twins of a twin pair are plated randomly, batch effects will partition into the unique environment variance component. The DBS plating layout had been established for a different study interested in differences between twin pairs in blood markers. The static associated with tiny blood spots meant that replating was not practical. However, the extensive technical controls implemented in the $25(\mathrm{OH}) \mathrm{D} 3$ assay eliminated plate effects and so confounding of plate and twin pair was not an issue.

\section{Statistical Analyses}

To account for non-normality of the data, a square root transformation was applied to TGF- $\beta 1$. Subsequently, TGF- $\beta 1$ and TNFR1 were standardized and effects of plate and year of blood sampling were regressed out. For $25(\mathrm{OH}) \mathrm{D} 3$, which is largely affected by seasonal differences in ultra violet exposure, a cosinor regression model was fitted to the data to adjust for seasonality; additionally, possible effects of sex, year of blood sampling, and paper type (Barnett \& Dobson, 2010) were regressed out. Year of blood sampling did not have a significant effect on any measure.

A saturated model was fitted to the residuals to estimate $\mathrm{MZ}$ and DZ twin correlations for cytokines TGF- $\beta 1$ and TNFR1 and for 25(OH)D3. A saturated model does not hold any assumptions regarding the underlying variance components model. Means and variances were constrained to be equal across zygosity groups, and twin correlations were constrained to be equal for same-sex DZ and oppositesex DZ twin pairs to improve stability of the genetic model. Within the saturated model, differences in means and variances between first- and second-born twins and between zygosity and sex (and BMI for 25(OH)D3) were tested using likelihood ratio tests.

To investigate the relative contributions of genetic and environmental factors to the observed variation in TGF- $\beta 1$, TNFR1, and 25(OH)D3, univariate variance components models were specified. The analysis of $25(\mathrm{OH}) \mathrm{D} 3$ was done in the full sample as well as separately, including individuals who had their blood collected in summer or winter (where the months, May to October inclusive were considered winter, and November to April inclusive were considered summer in the southern hemisphere). Within these models, the variance was partitioned into additive genetic (A), common environmental (C), and unique environmental (E) variance components. 'A' represents the additive genetic effects of alleles summed up over all genetic loci in the genome, ' $\mathrm{C}$ ' represents shared or common environmental factors that render offspring of the same family more alike, and ' $E$ ' represents environmental factors that result in differences between family members. 'E' also includes measurement error.

To examine the significance of estimated variance components, the fit of a nested (increasingly more restricted) model was compared to the fit of the full model. Two submodels were considered: (1) a model including A and E, and (2) a model including C and E. Goodness-of-fit of these submodels was assessed using likelihood ratio tests. The difference in log-likelihoods between the full (ACE) model and the reduced (AE or CE) model follows a $\chi^{2}$ distribution and was evaluated using a $\chi^{2}$-difference test. If the $\chi^{2}$-difference test is significant, the estimate of the variance component that is removed from the model (e.g., $\mathrm{C}$ in an AE model) is considered significantly larger than zero. If the $\chi^{2}$-difference test is not significant, the estimate of the variance component that is removed from the model is not significantly larger than zero. Twin correlations and variance components were estimated in the statistical software package Mx (Neale et al., 2006).

\section{Results}

Means and variances of raw values for TGF- $\beta 1$, TNFR1, and $25(\mathrm{OH}) \mathrm{D} 3$ are shown in Table 1 . For TGF- $\beta 1$ and TNFR1 we were not able to find an agreed normal reference range to which we could compare our results, vitamin D levels were within the normal range (Paxton et al., 2013). For TNFR1, we observed a significant difference in means between males and females, with males having higher levels than females $(p<.001)$. For $25(\mathrm{OH}) \mathrm{D} 3$, we observed significant differences in variances $(p=.001)$ between $M Z$ and DZ twins (with MZ twins having on average higher levels and larger variance compared with DZ twins) and a birth order effect in both means $(p<.05)$ and variances $(p<$ .01 ) (with first-born twins having on average lower levels of vitamin $\mathrm{D}$ and smaller variance). We expect the effects of zygosity and birth order to be a result of the sampling variance. The phenotypic correlations between the vitamin $\mathrm{D}$ and the two cytokine measures were not significantly different from zero.

MZ correlations were significantly higher than DZ correlations for TGF- $\beta 1$ and $25(\mathrm{OH}) \mathrm{D} 3$, and approached significance for TNFR1 (Table 1), suggesting a genetic component in the variance decomposition. Given the small sample size, 


\begin{tabular}{|c|c|c|c|c|}
\hline & Mean & Variance & $\mathrm{rMZ}(95 \% \mathrm{Cl})$ & $\mathrm{rDZ}(95 \% \mathrm{Cl})$ \\
\hline \multirow[t]{3}{*}{ TGF- $\beta 1$} & A: 30.54 & A: 327.75 & A: $0.72(0.62,0.80)$ & A: $0.45(0.29,0.58)$ \\
\hline & M: 29.66 & M: 339.30 & M: $0.68(0.50,0.79)$ & M: $0.60(0.30,0.76)$ \\
\hline & $F: 31.45$ & $F: 316.41$ & $F: 0.77(0.63,0.85)$ & $F: 0.54(0.31,0.70)$ \\
\hline \multirow[t]{3}{*}{ TNFR1 } & A: 809.25 & A: 85039.67 & A: $0.52(0.35,0.65)$ & A: $0.21(0.04,0.37)$ \\
\hline & M: 890.70 & M: 82972.81 & $\mathrm{M}: 0.53(0.23,0.70)$ & M: $0.27(0.00,0.48)$ \\
\hline & $F: 725.01$ & $F: 73712.50$ & $F: 0.52(0.29,0.67)$ & $F: 0.25(-0.10,0.52)$ \\
\hline \multirow[t]{3}{*}{$25(\mathrm{OH}) \mathrm{D} 3$} & A: 69.51 & A: 752.87 & A: $0.91(0.87,0.94)$ & A: $0.49(0.33,0.62)$ \\
\hline & M: 71.84 & M: 824.77 & M: $0.92(0.87,0.95)$ & M: $0.76(0.60,0.85)$ \\
\hline & $F: 67.16$ & $F: 673.56$ & F: $0.91(0.84,0.94)$ & $F: 0.71(0.49,0.82)$ \\
\hline
\end{tabular}

TABLE 2

Heritabilities of TGF- $\beta 1$, TNFR1, and 25(OH)D3 (with 95\% Confidence Intervals)

\begin{tabular}{lccr}
\hline & A & C & E \\
\hline TGF- $\beta 1$ & $0.57(0.26,0.80)$ & $0.16(0.00,0.42)$ & $0.27(0.20,0.38)$ \\
TNFR1 & $0.50(0.11,0.63)$ & $0.00(0.00,0.29)$ & $0.50(0.37,0.66)$ \\
25(OH)D3 - overall & $0.86(0.61,0.94)$ & $0.06(0.00,0.30)$ & $0.09(0.06,0.13)$ \\
25(OH)D3 - summer & $0.90(0.60,0.94)$ & $0.00(0.00,0.30)$ & $0.10(0.06,0.17)$ \\
25(OH)D3 - winter & $0.56(0.31,0.92)$ & $0.36(0.00,0.60)$ & $0.08(0.05,0.14)$ \\
\hline Note: $A=$ standardized additive genetic variance; $C=$ standardized common environmental variance; $E=$ standardized unique
\end{tabular}

we did not estimate heritability separately for males and females. Twin correlations, however, were very similar for males and females, suggesting no differences in heritability estimates across sex in our data (Table 1).

Sex was included as a covariate in the means model in the genetic analyses. We did not include sex as a covariate in the variance model, nor did we consider birth order effects in our genetic models. Including those effects in our genetic models would greatly reduce power given the current sample size, which in turn would probably lead to spurious results.

Within the genetic model, the two cytokines were found to be moderately heritable (Table 2), with heritabilities estimated at $0.57\left(95 \% \mathrm{CI} 0.26-0.80, \chi^{2}=12.49, p=<.001\right)$ and 0.50 (95\% CI $\left.0.11-0.63, \chi^{2}=5.9, p=.008\right)$ for TGF$\beta 1$ and TNFR1, respectively. Heritability of $25(\mathrm{OH}) \mathrm{D} 3$ (all months combined) was estimated to be high $(0.86,95 \%$ CI $0.61-0.94, \chi^{2}=55.98, p=<.001$ ) (Table 2). Heritability of $25(\mathrm{OH}) \mathrm{D} 3$ for individuals who had blood collected in the summer months was also high $(0.90,95 \%$ CI $0.60-$ $0.94, \chi^{2}=25.43, p=<.001$, while heritability for individuals who had blood collected in winter was moderate $(0.56$, $95 \%$ CI $\left.0.31-0.92, \chi^{2}=23.023, p=.001\right)$. For the cytokines TGF- $\beta 1$ and TNFR1, any plate or batch effects would tend to increase the unique environment variance component.

\section{Discussion}

We set out to determine if potential biomarkers for MDD and schizophrenia were heritable in a community cohort of healthy adolescent twins. Mean levels of circulating vitamin $\mathrm{D}$, measured as $25(\mathrm{OH}) \mathrm{D} 3$, were in the normal range for healthy Australian adolescents (Paxton et al., 2013). The differences in variances observed for $25(\mathrm{OH}) \mathrm{D} 3$ may have been due to chance. The heritability estimates of the two cytokines that could be measured in plasma were found to be moderate $(57 \%$ and $50 \%$ for TGF- $\beta 1$ and TNFR1, respectively) while the heritability of vitamin $\mathrm{D}$ was found to be high $(86 \%)$.

The heritability estimate for the standardized values of vitamin $\mathrm{D}$ was similar to the heritability of 0.80 found for vitamin D - specifically also 25(OH)D3 in the German Asthma Family Study Group (Wjst et al., 2007), and 0.86 found for $25(\mathrm{OH}) \mathrm{D}$ (as 25(OH)D3) in male rural Chinese adolescent twins (Arguelles et al., 2009) - but greater than the estimate of 0.43 estimated in adult twins from the St Thomas UK Adult Twin Registry (Hunter et al., 2001). Similar to Snellman et al. (2009), we found the heritability estimate of vitamin $D$ to be higher in the summer months. This was in contrast to another study, which observed heritability of vitamin $\mathrm{D}$ to be higher in winter months (Karohl et al., 2010). The only previous published report we could find estimating the heritability of TGF- $\beta 1$ found a heritability of 0.28 ; however, the levels of this cytokine were estimated in an antigen-stimulated whole-blood assay (Stein et al., 2003). We could not find published reports of heritabilities of TNFR1 to which to compare our results.

A genetic contribution to variation in circulating levels of cytokines TGF- $\beta 1$ and TNFR1 and vitamin D is potentially relevant in the etiology of MDD and schizophrenia, as 
each of these biomarkers may contribute to a role in the etiology of these disorders. Here we show the variance of these biomarkers is largely genetic, while changes in environmental factors appear to have less influence. Variants in the major histocompatibility complex (MHC) region have been associated with schizophrenia (Ripke et al., 2011; 2014), and many of the genes of this region code for cytokines (Shiina et al., 2009). This association does not appear to be driven by the high linkage disequilibrium in the extended MHC (Ripke et al., 2014). With the complexity of the MHC region presenting challenges in understanding its role in schizophrenia (Lehner, 2012), further study of shared genetic factors between these biomarkers and schizophrenia is warranted. The possibility of shared genetic factors between these biomarkers and MDD could also be investigated in future studies.

It should also be noted that although we did not find a strong positive phenotypic association between cytokines and vitamin D measures, this was to be expected. Stimulation of human CD4+CD25-T lymphocytes in the presence of $1,25(\mathrm{OH}) 2 \mathrm{D} 3$ has been shown to inhibit production of pro-inflammatory cytokines, including IFN- $\gamma$ and IL-17 (Jeffery et al., 2009). Low levels of plasma 25(OH)D3 have been associated with chronic disease where inflammation has been found to play an important role, such as type 2 diabetes (Ozfirat \& Chowdhury, 2010). This finding may not diminish the potential usefulness of these blood biomarkers, rather it suggests that the role of vitamin D deficiency in inflammation may be an area where further research is warranted (Yin \& Agrawal, 2014).

A limitation of our study is that healthy adolescents are expected to have very low levels of circulating inflammatory cytokines (Miller et al., 2011). This may explain why our initial ELISA multiplex assay performed on plasma samples was not sufficiently sensitive (i.e., did not generate usable results) to the circulating levels of cytokines in our healthy community cohort of adolescents. This was despite the assay being calibrated to standardized adult samples (McAfoose et al., 2009). To address the limitation of assay sensitivity, high-sensitivity assays (such as ELISA single assays or high sensitivity kits based on the Luminex 200 system) can be used. ELISA assays utilize undiluted samples, but they usually need high volume of sample (up to $500 \mu \mathrm{L}$ per sample). A limitation of the TGF- $\beta 1$ assay was the volume of sample required. The human TGF- $\beta 1$ Duoset ELISA used required $100 \mu$ l of sample. This was higher than the volume of aliquots available, so we decreased the sample volume used to $50 \mu \mathrm{l}$. In addition, the release of TGF- $\beta 1$ from platelet granules upon platelet activation also needs to be considered. Our protocol should remove platelets from the plasma sample but incomplete removal of platelets, which can cause variable and irreproducible results (Flislak et al., 2005), would serve to reduce estimates of heritability.

Recommendations for future studies could include extending this research to adolescents with MDD or schizophrenia (or psychotic symptoms) and conducting both univariate and bivariate analyses to investigate to what extent genetic impact on circulating levels of cytokines and vitamin $\mathrm{D}$ are heritable and whether the same genes affect these measures in people with psychiatric disorders such as MDD and schizophrenia. However, high sensitivity assays (such as ELISA single assays), with larger sample sizes would be required. This could be followed by investigating the role of other potential blood biomarkers (such as CRP and antibodies to infectious agents) in adolescents with MDD or psychosis.

\section{Conclusion}

This study reports heritability estimates of cytokines/inflammatory markers and vitamin $\mathrm{D}$ in a community-based cohort of adolescent twins. The heritability estimate for vitamin D was high, whereas the two cytokines that could be measured in plasma showed moderate heritabilities. As presented in the published literature, we found measurement of cytokines at the levels circulating in healthy adolescents to be difficult, perhaps limiting their utility. Further research (which takes into account the limitations of our present study) is warranted to explore the potential uses of these biomarkers, in particular in adolescents with mental health disorders.

\section{Ethical Standards}

The authors assert that all procedures contributing to this work comply with the ethical standards of the relevant national and institutional committees on human experimentation and with the Helsinki Declaration of 1975, as revised in 2008.

\section{Acknowledgments}

We thank the Brisbane twins and siblings for their participation; Marlene Grace and Ann Eldridge for sample collection; Kerrie McAloney for study coordination; Anthony Conciatore for IT support, Leanne Wallace and the Molecular Genetics Laboratory for sample preparation. The research was supported by Rotary Mental Health, the Australian Research Council (A7960034, A79906588, A79801419, DP0212016, DP0343921) and by the National Health and Medical Research Council $(389891,1049911,1069141)$.

\section{References}

Arguelles, L. M., Langman, C. B., Ariza, A. J., Ali, F. N., Dilley, K., Price, H., ... Wang, X. (2009). Heritability and environmental factors affecting vitamin D status in rural Chinese adolescent twins. Journal of Clinical Endocrinology \& Metabolism, 94, 3273-3281

Arias, I., Sorlozano, A., Villegas, E., de Dios Luna, J., McKenney, K., Cervilla, J., ... Gutierrez, J. (2012). Infectious agents associated with schizophrenia: A meta-analysis. Schizophrenia Research, 136, 128-136. 
Barnett, A. G., \& Dobson, A. J. (2010). Analysing seasonal health data. New York: Springer.

Benros, M. E., Mortensen, P. B., \& Eaton, W. W. (2012). Autoimmune diseases and infections as risk factors for schizophrenia. Neuroimmunomodulation in Health and Disease II, 1262, 56-66.

Bruce, D., Yu, S. H., Ooi, J. H., \& Cantorna, M. T. (2011). Converging pathways lead to overproduction of IL-17 in the absence of vitamin D signaling. International Immunology, $23,519-528$.

Cantorna, M. T. (2012). Vitamin D, multiple sclerosis and inflammatory bowel disease. Archives of Biochemistry and Biophysics, 523, 103-106.

Danese, A., Caspi, A., Williams, B., Ambler, A., Sugden, K., Mika, J., ... Arseneault, L. (2011). Biological embedding of stress through inflammation processes in childhood. Molecular Psychiatry, 16, 244-246.

Danese, A., Pariante, C. M., Caspi, A., Taylor, A., \& Poulton, R. (2007). Childhood maltreatment predicts adult inflammation in a life-course study. Proceedings of the National Academy of Sciences of the United States of America, 104, 1319-1324.

Dowlati, Y., Herrman, N., Swardfager, W., Liu, H., Sham, L., Reim, E. K., .. Lanctot, K. L. (2010). A meta-analysis of cytokines in major depression. Biological Psychiatry, 67, 446-457.

Eyles, D., Anderson, C., Ko, P., Jones, A., Thomas, A., Burne, T., ... McGrath, J. (2009). A sensitive LC/MS/MS assay of $250 \mathrm{H}$ vitamin D-3 and $250 \mathrm{H}$ vitamin D-2 in dried blood spots. Clinica Chimica Acta, 403, 145-151.

Flislak, R., Jaroszewicz, J., Lapinski, T. W., Flislak, I., Rogalska, M., \& Prokopowicz, D. (2005). Plasma transforming growth factor beta(1), metalloproteinase-1 and tissue inhibitor of metalloproteinases-1 in acute viral hepatitis type B. Regulatory Peptides, 131, 54-58.

Gabbay, V., Klein, R. G., Alonso, C. M., Babb, J. S., Nishawala, M., De Jesus, G., ... Gonzalez, C. J. (2009). Immune system dysregulation in adolescent major depressive disorder. Journal of Affective Disorders, 115, 177-182.

Garber, J., \& Weersing, V. R. (2010). Comorbidity of anxiety and depression in youth: Implications for treatment and prevention. Clinical Psychology Science and Practice, 17, 293-306.

Glowinski, A. L., Madden, P. A. F., Bucholz, K. K., Lynskey, M. T., \& Heath, A. C. (2003). Genetic epidemiology of selfreported lifetime DSM-IV major depressive disorder in a population-based twin sample of female adolescents. Journal of Child Psychology and Psychiatry and Allied Disciplines, 44, 988-996.

Gressner, A. M., Weiskirchen, R., Breitkopf, K., \& Dooley, S. (2002). Roles of TGF-beta in hepatic fibrosis. Frontiers in Bioscience, 7, D793-D807.

Hoang, M. T., DeFina, L. F., Willis, B. L., Leonard, D. S., Weiner, M. F., \& Brown, E. S. (2011). Association between low serum 25-hydroxyvitamin D and depression in a large sample of healthy adults: The Cooper center longitudinal study. Mayo Clinic Proceedings, 86, 1,0501,055 .
Holick, M. F. (2007). Vitamin D deficiency. New England Journal of Medicine, 357, 266-281.

Howren, M., Lamkin, D., \& Suls, J. (2009). Associations of depression with C-reactive protein, IL-1, and IL-6: A metaanalysis. Psychosomatic Medicine, 71, 171-186.

Hunter, D., De Lange, M., Snieder, H., MacGregor, A. J., Swaminathan, R., Thakker, R. V., ... Spector, T. D. (2001). Genetic contribution to bone metabolism, calcium excretion, and vitamin $\mathrm{D}$ and parathyroid hormone regulation. Journal of Bone and Mineral Research, 16, 371-378

Irwin, M., \& Miller, A. (2007). Depressive disorders and immunity: 20 years of progress and discovery. Brain, Behavior, and Immunity, 21, 374-383.

Janssen, D., Caniato, R., Verster, J., \& Baune, B. (2010). A psychoneuroimmunological review on cytokines involved in antidepressant treatment response. Human Psychopharmacology-Clinical and Experimental, 25, 1-15.

Jeffery, L. E., Burke, F., Mura, M., Zheng, Y., Qureshi, O. S., Hewison, M., ... Sansom, D. M. (2009). 1,25Dihydroxyvitamin D-3 and IL-2 combine to inhibit T cell production of inflammatory cytokines and promote development of regulatory T cells expressing CTLA-4 and FoxP3. Journal of Immunology, 183, 5458-5467.

Kapur, S., Phillips, A. G., \& Insel, T. R. (2012). Why has it taken so long for biological psychiatry to develop clinical tests and what to do about it? Molecular Psychiatry, 17, 11741179.

Karohl, C., Su, S. Y., Kumari, M., Tangpricha, V., Veledar, E., Vaccarino, V., ... Raggi, P. (2010). Heritability and seasonal variability of vitamin D concentrations in male twins. American Journal of Clinical Nutrition, 92, 1393-1398.

Kendler, K. S. (2013). What psychiatric genetics has taught us about the nature of psychiatric illness and what is left to learn. Molecular Psychiatry, 18, 1058-1066.

Kvaskoff, D., Ko, P., Simila, H. A., \& Eyles, D. W. (2012). Distribution of 25-hydroxyvitamin D-3 in dried blood spots and implications for its quantitation by tandem mass spectrometry. Journal of Chromatography B-Analytical Technologies in the Biomedical and Life Sciences, 901, 47-52.

Lehner, T. (2012). The genes in the major histocompatibility complex as risk factors for schizophrenia: De omnibus dubitandum. Biological Psychiatry, 72, 615-616.

McAfoose, J., Koerner, H., \& Baune, B. T. (2009). The effects of TNF deficiency on age-related cognitive performance. Psychoneuroendocrinology, 34, 615-619.

McGrath, J. J., Eyles, D. W., Pedersen, C. B., Anderson, C., Ko, P., Burne, T. H., ... Mortensen, P. B. (2010). Neonatal vitamin D status and risk of schizophrenia: A population-based case-control study. Archives of General Psychiatry, 67, 889894.

Miller, B. J., Buckley, P., Seabolt, W., Mellor, A., \& Kirkpatrick, B. (2011). Meta-analysis of cytokine alterations in schizophrenia: Clinical status and antipsychotic effects. $B i-$ ological Psychiatry, 70, 663-671.

Mills, N. T., Scott, J. G., Wray, N. R., Cohen-Woods, S., \& Baune, B. T. (2013). Research review: The role of cytokines in depression in adolescents: A systematic review. The Journal of Child Psychology and Psychiatry, 54, 816-835. 
Neale, M., Boker, S., Xie, G., \& Maes, H. (2006). Mx: Statistical modeling (7th ed.). Richmond, VA: Department of Psychiatry, Virginia Commonwealth University.

O’Brien, S. M., Fitzgerald, P., Scully, P., Landers, A. M., Scott, L. V., \& Dinan, T. G. (2007). Impact of gender and menstrual cycle phase on plasma cytokine concentrations. Neuroimmunomodulation, 14, 84-90.

Ozfirat, Z., \& Chowdhury, T. A. (2010). Vitamin D deficiency and type 2 diabetes. Postgraduate Medical Journal, 86, 1825.

Palm, S., Postler, E., Hinrichsen, H., Maier, H., Zabel, P., \& Kirch, W. (1996). Twenty-four-hour analysis of lymphocyte subpopulations and cytokines in healthy subjects. Chronobiology International, 13, 423-434.

Paxton, G. A., Teale, G. R., Nowson, C. A., Mason, R. S., McGrath, J. J., Thompson, M. J., ... Munns, C. F. (2013). Vitamin D and health in pregnancy, infants, children and adolescents in Australia and New Zealand: A position statement. Medical Journal of Australia, 198, 142-143.

Posthuma, D., Meulenbelt, I., de Craen, A. J. M., de Geus, E. J. C., Slagboom, P. E., Boomsma, D. I., ... Westendorp, R. G. J. (2005). Human cytokine response to ex vivo amyloid-beta stimulation is mediated by genetic factors. Twin Research and Human Genetics, 8, 132-137.

Ripke, S., Neale, B. M., Corvin, A., Walters, J. T. R., Farh, K. H., Holmans, P. A., ... Wellcome Trust Case-Control Consortium (2014). Biological insights from 108 schizophreniaassociated genetic loci. Nature, 511, 421-427.

Ripke, S., Sanders, A. R., Kendler, K. S., Levinson, D. F., Sklar, P., Holmans, P. A., ... Schizophrenia Psychiatric Genome-Wide Association Study (GWAS) Consortium (2011). Genome-wide association study identifies five new schizophrenia loci. Nature Genetics, 43, 969-977.

Sas, A. A., Jamshidi, Y., Zheng, D., Wu, T., Korf, J., Alizadeh, B. Z., ... Snieder, H. (2012). The age-dependency of genetic and environmental influences on serum cytokine levels: A twin study. Cytokine, 60, 108-113.

Schwab, S. G., \& Wildenauer, D. B. (2013). Genetics of psychiatric disorders in the GWAS era: An update on schizophrenia. European Archives of Psychiatry and Clinical Neuroscience, 263, S147-S154.

Shiina, T., Hosomichi, K., Inoko, H., \& Kulski, J. K. (2009). The HLA genomic loci map: Expression, interaction, diversity and disease. Journal of Human Genetics, 54, 15-39.

Skogstrand, K. (2012). Multiplex assays of inflammatory markers, a description of methods and discussion of precautions - Our experience through the last ten years. Methods, 56, 204-212.

Skogstrand, K., Ekelund, C. K., Thorsen, P., Vogel, I., Jacobsson, B., Norgaard-Pedersen, B., .. . Hougaard, D. M.
(2008). Effects of blood sample handling procedures on measurable inflammatory markers in plasma, serum and dried blood spot samples. Journal of Immunological Methods, 336, 78-84.

Skogstrand, K., Thorsen, P., Norgaard-Pedersen, B., Schendel, D. E., Sorensen, L. C., \& Hougaard, D. M. (2005). Simultaneous measurement of 25 inflammatory markers and neurotrophins in neonatal dried blood spots by immunoassay with xMAP technology. Clinical Chemistry, 51, 18541866.

Snellman, G., Melhus, H., Gedeborg, R., Olofsson, S., Wolk, A., Pedersen, N. L., ... Michaelsson, K. (2009). Seasonal genetic influence on serum 25-hydroxyvitamin D levels: A twin study. PLoS One, 4, e7747.

Stein, C. M., Guwatudde, D., Nakakeeto, M., Peters, P., Elston, R. C., Tiwari, H. K., ... Whalen, C. C. (2003). Heritability analysis of cytokines as intermediate phenotypes of tuberculosis. Journal of Infectious Diseases, 187, 1679-1685.

Sullivan, P. F., Neale, M. C., \& Kendler, K. S. (2000). Genetic epidemiology of major depression: Review and metaanalysis. American Journal of Psychiatry, 157, 1552-1562.

Tolppanen, A.-M., Sayers, A., Fraser, W. D., Lewis, G., Zammit, S., \& Lawlor, D. A. (2012). The association of serum 25hydroxyvitamin D3 and D2 with depressive symptoms in childhood - A prospective cohort study. Journal of Child Psychology and Psychiatry, 53, 757-766.

Veldman, C. M., Cantorna, M. T., \& DeLuca, H. F. (2000). Expression of 1,25-dihydroxyvitamin D-3 receptor in the immune system. Archives of Biochemistry and Biophysics, 374, 334-338.

Wahl, S. M. (1992). Transforming growth-factor-beta (TGFBeta) in inflammation - A cause and a cure. Journal of Clinical Immunology, 12, 61-74.

Wajant, H., Pfizenmaier, K., \& Scheurich, P. (2003). Review: Tumor necrosis factor signaling. Cell Death and Differentiation, 10, 45-65.

Wjst, M., Altmueller, J., Braig, C., Bahnweg, M., \& Andre, E. (2007). A genome-wide linkage scan for 25-OH-D-3 and $1,25-(\mathrm{OH})(2)-\mathrm{D}-3$ serum levels in asthma families. Journal of Steroid Biochemistry and Molecular Biology, 103, 799-802.

Wright, M. J., \& Martin, N. G. (2004). Brisbane adolescent twin study: Outline of study methods and research projects. Australian Journal of Psychology, 56, 65-78.

Yin, K., \& Agrawal, D. K. (2014). Vitamin D and inflammatory diseases. Journal of Inflammation Research, 7, 69-87.

Yolken, R. H., Torrey, E. F., Lieberman, J. A., Yang, S., \& Dickerson, F. B. (2011). Serological evidence of exposure to herpes simplex virus type 1 is associated with cognitive deficits in the CATIE schizophrenia sample. Schizophrenia Research, 128, 61-65. 